\title{
Topological properties of graph of hydrogen bonds forming in SOD1 protein indicate critical regions in its structure
}

\author{
A. Alemasov*, V. Ivanisenko \\ Institute of Cytology and Genetics SB RAS, Novosibirsk, Russia \\ *e-mail:alemasov@bionet.nsc.ru
}

Key words: ALS, SOD1, hydrogen bonds, elastic network models, graph theory

Motivation and Aim: Amyotrophic lateral sclerosis (ALS) is a fatal neurodegenerative disease which manifests in two forms: familial and sporadic [1]. The second most prevalent and studied cause of familial ALS is mutations in the SOD1 gene, which codes for the superoxide dismutase- 1 enzyme. One of hypotheses on the mechanism for disease progression posits an aggregation of misfolded SOD1 proteins caused by mutations.

Methods and Algorithms: Spatial structures of the 35 mutant SOD1s were obtained by introduction of mutations into the human WT SOD1 protein structure (PDBID: 2V0A) using FoldX software. The WT and 35 mutant structures were modelled with elastic networks modelling $(\mathrm{EN})$ using EINemo with the default parameters set. A table of hydrogen bonds was constructed for the WT SOD1 and its mutants by the cpptraj utility using AmberTools 13. Rigid fragments in hydrogen bonds graph were detected by starfish [2]. A topology of the graph was constructed using nested stochastic block model from Python's graph-tool package. Graph vertices whose remove decreases connected component size in the graph were found with Python's networkx package.

Results: Mutant SOD1' residues being bottleneck vertices in the graph were found to form hydrogen bonds lacking in the WT. This can indicate that mutations in SOD1 modify hydrogen bonds network to weaker one in terms of the network connectivity. Critical regions are found inside the protein structure which are sensitive for structural adjustments and were affected by ALS-linked mutations. These protein structure regions include amino-acid residues from zinc-binding site, zinc-bindnig and electrostatic loop. Conclusion: The results of graph analysis are in agreement with our previous work [3] in those SOD1 regions which are critical for its structure if affected by ALS mutations. These SOD1 residues identified can be responsible for the pathogenic conformations in the mutants. Taking them into account can be valuable both for understanding the molecular mechanisms of mutant pathogenicity and drug design. The results garnered in this study can aid the design of drugs that not only can prevent aggregate formation, but also repair pathogenic conformations of mutant proteins before aggregation.

Acknowledgements: Methods development was supported by the RFBR grant No. 17-54-49004 and critical regions study was supported by the Russian Science Foundation grant No. 14-44-00011.

\section{References}

1. Kiernan M.C. et al. (2011) Amyotrophic lateral sclerosis. Lancet. 377:942-955.

2. Duggal G., Kingsford C. (2012) Graph rigidity reveals well-constrained regions of chromosome conformation embeddings. BMC Bioinformatics. 13(1):241.

3. Alemasov N.A. et al. (2018). Molecular mechanisms underlying the impact of mutations in SOD1 on its conformational properties associated with amyotrophic lateral sclerosis as revealed with molecular modelling. BMC Structural Biology. 18(1):1. 